\title{
Surface electron perturbations and the collective behaviour of atoms adsorbed on a cylinder
}

\author{
Boris Dzyubenko, Hao-Chun Lee, Oscar E. Vilches and David H. Cobden ${ }^{\star}$
}

A single-walled carbon nanotube presents a seamless cylindrical graphene surface and is thus an ideal adsorption substrate for investigating the physics of atoms and molecules in two dimensions and approaching the one-dimensional limit ${ }^{1-7}$. When a suspended nanotube is made into a transistor, frequency shifts of its mechanical resonances allow precise measurement of the adsorbed mass down to the single-atom level $^{8-10}$. Here we show that its electrical characteristics are also modified by the adsorbed atoms and molecules, partly as a result of a small charge transfer between them and the carbon surface. We quantify this charge transfer, finding it similar for many different species, and use the associated sensitivity of the conductance to carry out the studies of phase transitions, critical scaling, dynamical fluctuations and dissipative metastable states in a system of interacting atoms confined to a cylindrical geometry.

Graphite is the substrate of choice for investigating the collective two-dimensional (2D) behaviour of adsorbed atoms and molecules, including transitions between $2 \mathrm{D}$ solid (S), liquid (L) and vapour (V) phases $^{11,12}$. In addition, the physical interactions of adsorbates with carbon are important in filters, electrodes, sensors and gas storage, but very little is known experimentally about their effects on the electrons in the surface. Using the unique combination of assets of suspended nanotube transistors, held in equilibrium vapour ${ }^{6}$, we are able to detect the charge transfer from neutral atoms or molecules. Surprisingly, it is of a similar magnitude for all the simple gases tested $\left({ }^{4} \mathrm{He}, \mathrm{Ar}, \mathrm{Kr}, \mathrm{Xe}, \mathrm{N}_{2}, \mathrm{CO}\right.$ and $\left.\mathrm{O}_{2}\right)$, and although small (much less than predicted ${ }^{13-15}$ ), at gate voltages near threshold it can produce a large change in conductance. Thus, simply by monitoring the conductance we are able to explore the phase transitions of atoms on a cylinder, seeing $2 \mathrm{D}$ critical and triple points and critical behaviour matching the 2D Ising universality class with a finite-size cutoff. We also observe intriguing features in the phase transition dynamics, and discover nonlinear effects of adsorbates interacting with electrical current.

Each device, containing a nanotube of diameter $\sim 2 \mathrm{~nm}$ and suspended length $\sim 1 \mu \mathrm{m}$, is mounted in a vapour cell at temperature $T$ and pressure $P$, the latter being deduced from the pressure $P_{\mathrm{g}}$ on an external gauge, as indicated in Fig. 1a (see also Supplementary Information 1). The nanotubes have small bandgaps, producing a minimum in the conductance $G$ near zero gate voltage ${ }^{16} V_{\mathrm{g}}$, as can be seen in the characteristics of a device (YB11) shown in Fig. 1b. At lower temperatures, contact barriers cause the nanotube to act as a single-electron transistor exhibiting reproducible Coulomb blockade (CB) oscillations, visible in the characteristic at $4.3 \mathrm{~K}$ (blue).

Using the arrangement indicated in Fig. 1a, while measuring the conductance we can also detect mechanical resonances and deduce the coverage, $\phi$, which is the number of adsorbates per carbon atom (see Methods). For each gas, as the pressure is increased we find that a single dense monolayer forms before the vapour saturates.
During this process the $G-V_{\mathrm{g}}$ characteristics change slightly: for example, Fig. $1 \mathrm{~b}$ shows characteristics of YB11 at $47 \mathrm{~K}$ in vacuum (black) and in saturated argon (red). The change in $G$ is generally a few percent, and varies between devices (see Supplementary Information 2 for more examples), but it always has one common feature: a distinct shift of the 'gap' along the $V_{\mathrm{g}}$ axis, by an amount $\Delta V_{\mathrm{g}}$. This shift is usually most apparent and unambiguous at the $\mathrm{p}$ channel threshold, which tends to be sharper. However, in the case of helium we can see that the CB peaks shift roughly uniformly over the entire gate voltage range, as illustrated in Fig. 1c for another device, YB14. Such behaviour is not consistent with a change in capacitance, or in the contact transparency, but rather implies that a net voltage-independent charge is donated by the adsorbates to the nanotube, presumably associated with the hybridization of the adsorbate's orbitals with the surface electron states. From the period $\Delta V_{\mathrm{CB}}$ of the $\mathrm{CB}$ oscillations, which corresponds to adding the charge $-e$ of one electron, we can directly convert $\Delta V_{\mathrm{g}}$ to a change $\Delta Q=+e \Delta V_{\mathrm{g}} / \Delta V_{\mathrm{CB}}$ in the total charge on the nanotube.

Figure $1 \mathrm{~d}$ shows how $\Delta V_{\mathrm{g}}$ changes with argon coverage on device YB11 at several temperatures. In the limit of dense coverage $(\phi \sim 0.15), \Delta V_{\mathrm{g}}$ approaches $\sim+10 \mathrm{mV}$, independent of $T$. With $\Delta V_{\mathrm{CB}}=11 \mathrm{mV}$ this gives $\Delta Q \approx+0.9 e$, amounting to an average charge transfer per Ar atom of $\Delta Q / N_{\mathrm{A}} \sim+2 \times 10^{-5} e$, where $N_{\mathrm{A}}=\phi N_{\mathrm{C}} \sim 5 \times 10^{4}$ is the number of argon atoms and $N_{\mathrm{C}} \sim 2.5 \times 10^{5}$ the number of carbon atoms in the suspended part of the nanotube. In the limit of sparse coverage, however, $\Delta V_{\mathrm{g}}$ is approximately proportional to $\phi$, and we can obtain an average charge donated per atom from the slope. It is negative and grows in magnitude with decreasing $T$, reaching about $-7 \times 10^{-4} e$ at $42 \mathrm{~K}$. Both limits are plotted together in Fig. 1e. This behaviour is fairly typical, but there are large variations between devices in the sparse coverage limit. For example, with device YB14 there is no apparent negative swing and the $\mathrm{CB}$ peak shift is monotonic in helium pressure, as shown in Fig. 1f, although slightly different for the p-channel and n-channel peaks.

Close to threshold the change in $G$ at fixed gate voltage can be large, allowing it to be measured much more precisely than $\Delta V_{\mathrm{g}}$. In Fig. 2a we plot both $G$ and $\phi$ at a series of equilibrium pressures at $47 \mathrm{~K}$ for device YB30. Importantly, the upward swing in $G$ matches the riser in $\phi$, implying that most of the change in $G$ is a result of adsorption on the vibrating part of the nanotube, whatever the mechanism. The decrease in $G$ preceding the riser also tracks the variation of $\phi$, but with the opposite sign. The inset shows $G$ versus $\phi$, which is approximately linear at low density (red line), as expected if in this limit the contribution of each argon atom is independent.

The variation of $G$ with $P$ at fixed $T$ can be measured conveniently by gradually increasing the pressure, at the expense of some accuracy in $P$. Figure $2 \mathrm{~b}$ compares 'conductance isotherms' obtained in this way for $\mathrm{Ar}, \mathrm{CO}$ and $\mathrm{N}_{2}$ at $50 \mathrm{~K}$ on device YB27, and Fig. 2c compares $\mathrm{Ar}$ and $\mathrm{O}_{2}$ at different temperatures 
a

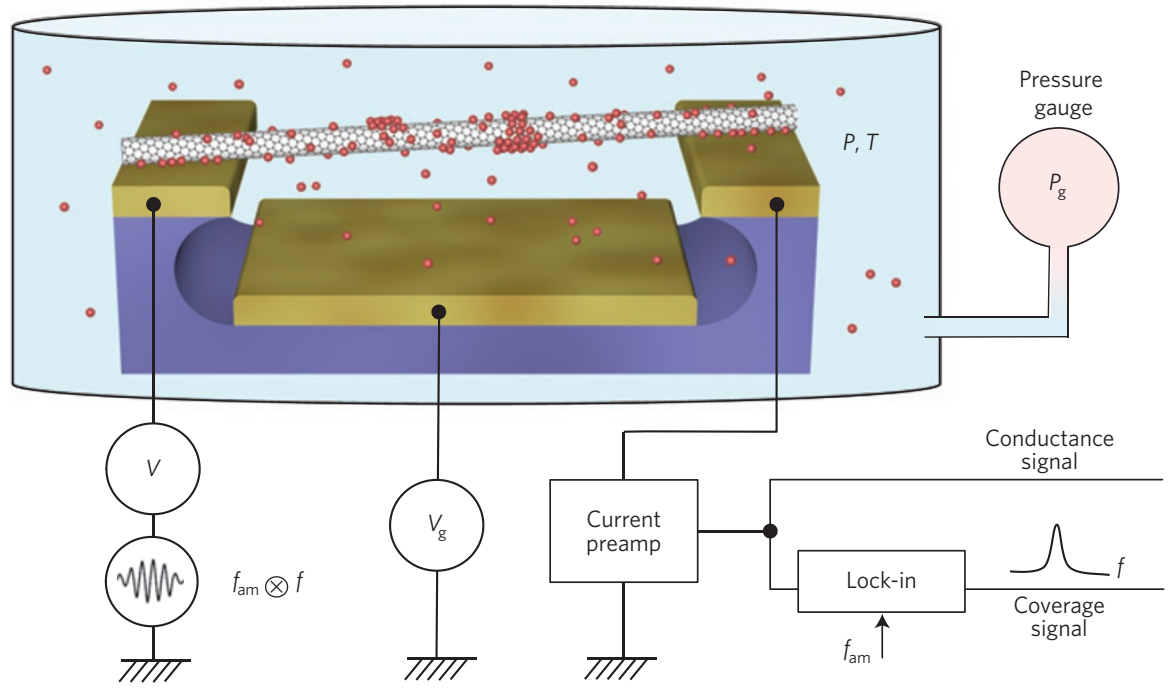

b

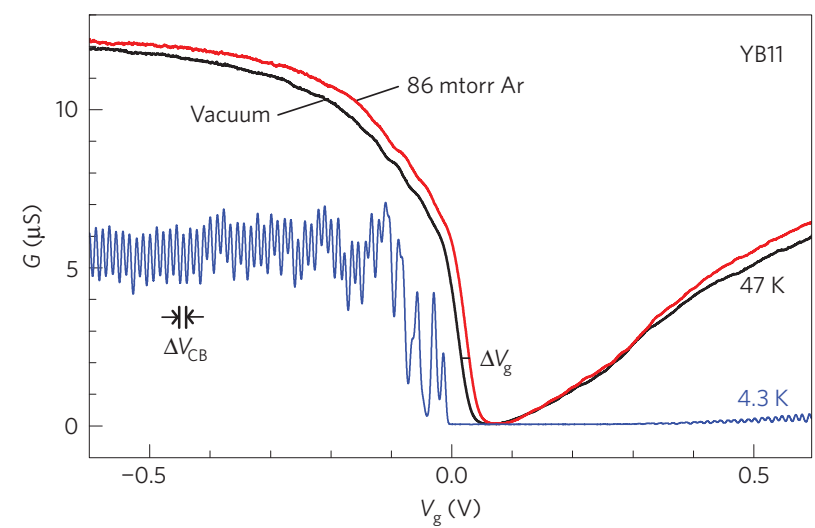

c

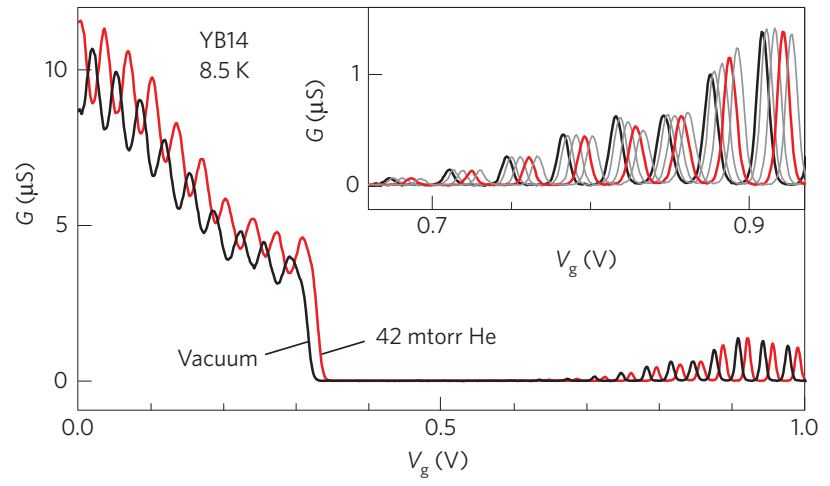

d

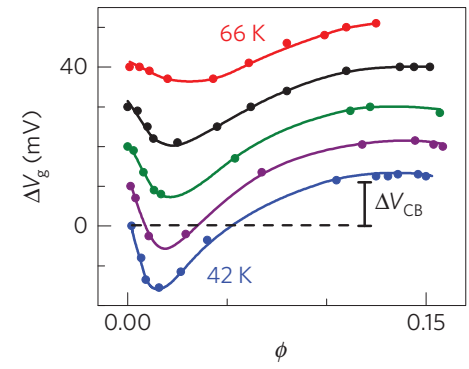

e

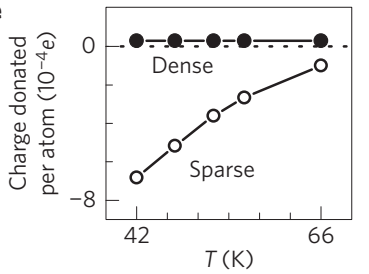

f

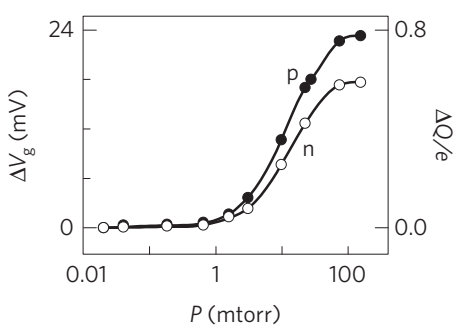

Figure 1 | Effects of adsorbates on nanotube conductance. a, Schematic arrangement for measuring both the conductance, $G$, and the coverage, $\phi$, (using a vibrational frequency shift) of a suspended nanotube in a vapour cell at temperature $T$ and pressure $P$ connected to an external gauge reading pressure $P_{\mathrm{g}}$. b. Characteristics of device YB11: in vacuum (black) and coated with a monolayer of $\mathrm{Ar}$ (red, $P_{\mathrm{g}}=86 \mathrm{mtorr}$ ) at $47 \mathrm{~K}$, showing a threshold shift $\Delta V_{\mathrm{g}}$; and in vacuum at $4.3 \mathrm{~K}$ (blue) showing Coulomb blockade oscillations with period $\Delta V_{\mathrm{CB}}$. c. Effect of ${ }^{4} \mathrm{He}$ on device $\mathrm{YB} 14$ at $8.5 \mathrm{~K}$. Inset: traces at 0.1 (black), 8 (grey), 21 (grey), 42 (red) and 220 (grey) mtorr, showing a steady, reproducible shift. $\mathbf{d}$, Threshold shift versus coverage at temperatures $T=42,47,52$, 56 and $66 \mathrm{~K}$ (offset sequentially by $10 \mathrm{mV}$ for clarity). Smooth curves are guides for the eye. e, Approximate charge transferred to the nanotube per Ar atom versus $T$ at sparse (open circles) and dense (solid circles) monolayer coverage. f, Shifts versus ${ }^{4}$ He pressure for the $p$-channel (solid) and $n$-channel (open circles) peaks in c.

on device YB24. We see that the behaviour of all these gases is strikingly similar, implying that their hybridization with the nanotube electrons is also very similar. Although this might at first seem surprising, it is consistent with the known fact that they have similar physisorption binding energies on graphite ${ }^{12}$. All are compact molecules/atoms of similar mass with stable electronic configurations and low polarizability, and our results suggest that the physics of the binding is quantitatively generic to such cases.

The effect varies between gases, but with the following common features (see Supplementary Information 2). First, at dense coverage $(\phi \approx 0.15)$ there is a charge transfer to the nanotube of the order of $e$ in total, or around $10^{-5} \mathrm{e}$ per molecule, which is almost independent 

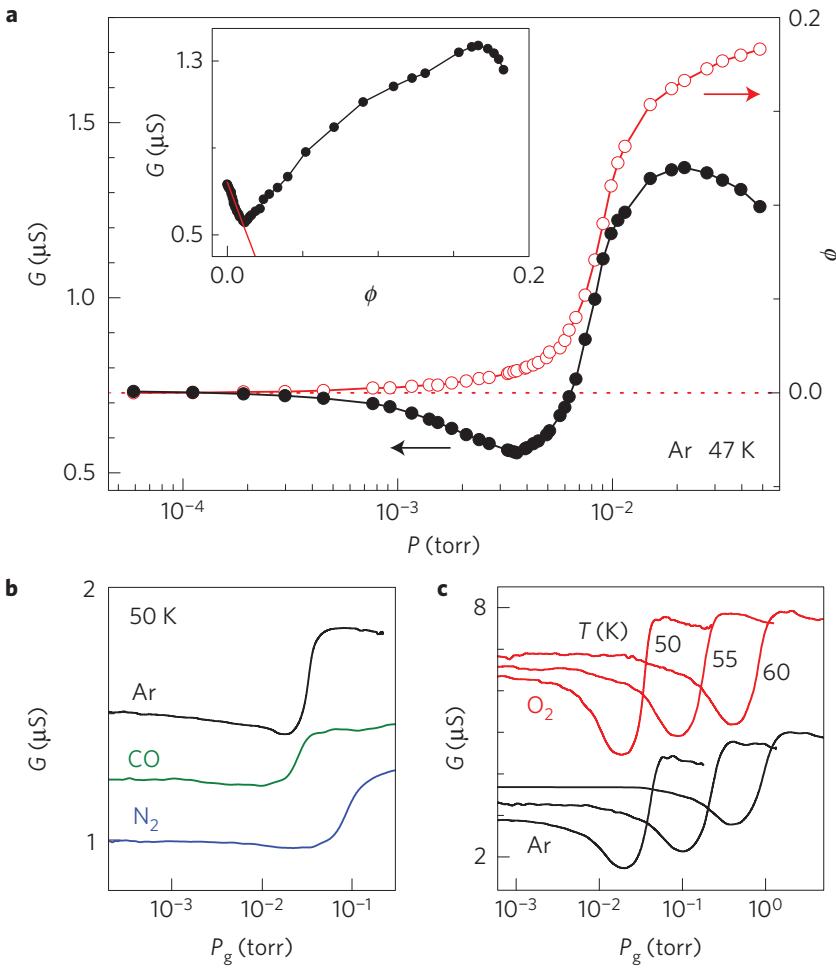

Figure 2 | Conductance isotherms. a, Coverage, $\phi$, (open red circles, right axis) and conductance, G, (solid black circles, left axis) measured together at a series of equilibrium Ar pressures at $47 \mathrm{~K}$ (device YB30,

$V_{g}=-0.05 \mathrm{~V}$ ). Inset: $G$ versus $\phi$, with low-density linear region indicated by a red line. $\mathbf{b}$, Continuous conductance isotherms at $50 \mathrm{~K}$ for $\mathrm{Ar}, \mathrm{CO}$ and $\mathrm{N}_{2}$ (device YB27; $V_{g}=-1.0 \mathrm{~V}$; upper traces offset for clarity). c, Comparison of $\mathrm{Ar}$ and $\mathrm{O}_{2}$ conductance isotherms at several temperatures (device YB24; $V_{g}=-0.5 \mathrm{~V} ; \mathrm{O}_{2}$ traces offset for clarity).

of temperature. For $\mathrm{Ar}, \Delta Q$ ranged from $+0.25 e$ to $+0.9 e$; for $\mathrm{N}_{2}$, from $-0.4 e$ to $+0.2 e$. Second, the charge transfer (as determined by $\mathrm{CB}$ shifts) is almost independent of gate voltage, and thus of a large electric field approaching $1 \mathrm{~V} \mathrm{~nm}^{-1}$ normal to the nanotube surface. Third, the change in conductance away from the gap on forming a monolayer is usually only a few percent, implying that the backscattering of electrons from adsorbates is very weak. Last, at sparse coverage the charge transfer is often of the opposite sign, grows as $T$ decreases, and varies in magnitude, from undetectable (in device YB14) to about $-10^{-3}$ e per molecule (in YB11). One factor in explaining this may be that although some nanotubes, such as YB14, can be deduced to be extremely clean from the phase transitions discussed below, others such as YB11 may have patches of contamination or amorphous carbon. The first adsorbates will attach to higher-binding sites surrounding the patches, where they might also induce a different charge. However, the amount of contamination must be small enough not to produce detectable features in the low-pressure portion of the coverage isotherms. In any case it is clear that the large responses to simple gases previously reported for nanotube $e^{17-20}$ and graphene $e^{21,22}$ devices were not associated with the clean surface but with contacts, substrate, contamination, or defects ${ }^{23,24}$, and our results provide a new improved basis for theoretical understanding of adsorption on carbon substrates.

We can now exploit the conductance as a powerful probe of the adsorbate system. It can be sensitive to changes in ordering as well as density; it does not rely on locating a vibrational resonance; and it can be measured with high precision in linear response, so minimally perturbing the system. For the device held near threshold in Fig. $2 \mathrm{a}$, changing $N_{\mathrm{A}}$ by one at small $\phi$ corresponds to a
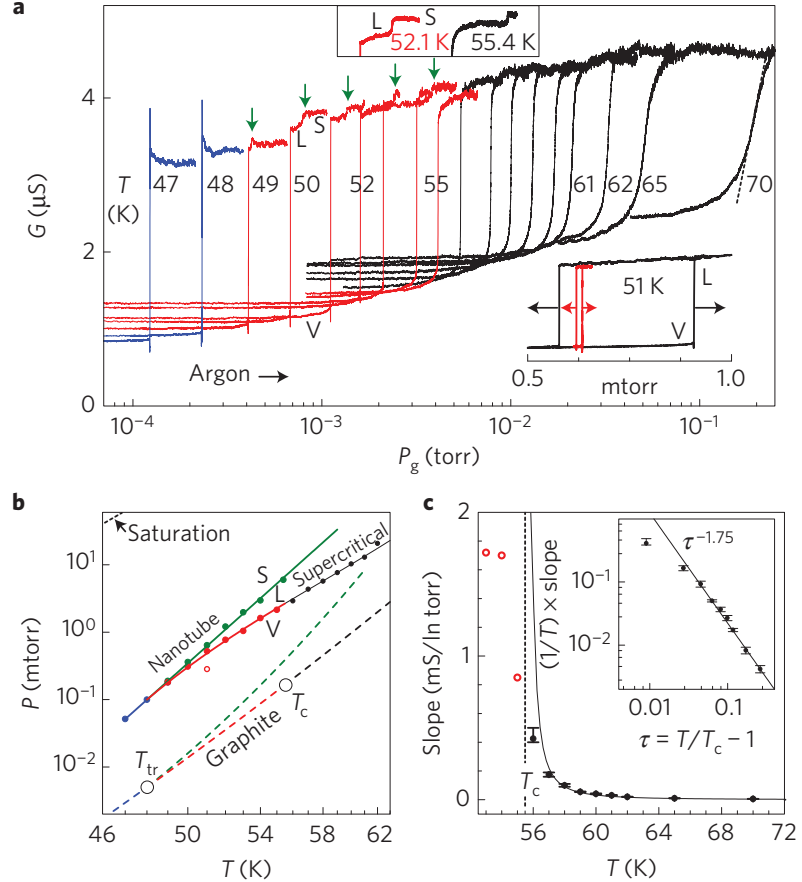

Figure 3 | Probing the phase boundaries and critical behaviour of two-dimensional argon. $\mathbf{a}$, Series of conductance isotherms each taken with Ar pressure increasing (device $Y B 14, V_{g}=0.34 \mathrm{~V}$ ). The interval is $1 \mathrm{~K}$ below $62 \mathrm{~K}$. Blue traces are below the inferred triple point, $T_{\mathrm{tr}} \approx 48 \mathrm{~K}$; red traces are between $T_{\mathrm{tr}}$ and the critical point, $T_{\mathrm{c}} \approx 55.5 \mathrm{~K}$; and black traces are supercritical. Upper inset: sections of slower isotherms showing the smaller riser (indicated by green arrows) more clearly. Lower inset: hysteresis caused by the cell pressure lag at $20 \mathrm{mtorr} \mathrm{h}^{-1}$ (black) and $1 \mathrm{mtorr} \mathrm{h}^{-1}$ (purple). b, Phase boundaries between 2D vapour (V), liquid ( $\mathrm{L}$ ) and solid (S) Ar on this nanotube deduced from the measurements in $\mathbf{a}$. Red and blue points are the positions of the larger riser, and green points of the small riser. The extra point (red open circle) at $51 \mathrm{~K}$ has no gauge lag error. Dashed lines are the corresponding 2D phase boundaries on graphite. c, Maximum slope of the main riser versus temperature. Error bars show range of straight line fits consistent with the data. Measurements below $T_{c}$ (red open circles) are limited by the system response. Inset: log-log plot of $(1 / T) \times($ slope) versus reduced temperature $\tau$. The line indicates the inverse power $\gamma=1.75$ for the $2 \mathrm{D}$ Ising universality class.

a measurable fractional change in $G$ of $\sim 10^{-3}$ (although we would expect $N_{\mathrm{A}}$ to fluctuate in equilibrium on a microsecond timescale). This sensitivity permits investigation of the collective behaviour of $2 \mathrm{D}$ matter on a cylinder. Recent simulations have predicted commensuration effects on the phases on the cylindrical nanotube surface $^{4}$, but for simplicity we focus here on argon, which is less likely to exhibit commensurate solid phases.

For Ar on graphite, the $2 \mathrm{D}$ triple point and critical points ${ }^{25,26}$ are $T_{\text {tr }} \approx 48 \mathrm{~K}$ and $T_{\mathrm{c}} \approx 55 \mathrm{~K}$, both lower than the $3 \mathrm{D}$ equivalents, with uncertainty of about $\pm 0.5 \mathrm{~K}$ in each (see Supplementary Information 3). Some nanotube devices, including YB14, show features matching these, implying that $\mathrm{Ar}$ on a clean nanotube surface forms a homogeneous quasi-2D system in which the interactions between atoms are the same as on graphite. Figure 3a shows conductance isotherms for Ar on YB14. The main riser becomes vertical below about $55 \mathrm{~K}$ (red traces), as expected for a first-order $\mathrm{V}-\mathrm{L}$ transition emerging below $T_{c}$, with continuous supercritical behaviour at higher temperatures (black traces). A small additional riser (green arrows), which is easier to identify in very slow pressure sweeps (upper inset) and disappears below $48 \mathrm{~K}$ (blue traces), indicates an L-S transition occurring above $T_{\mathrm{tr}}$. The 

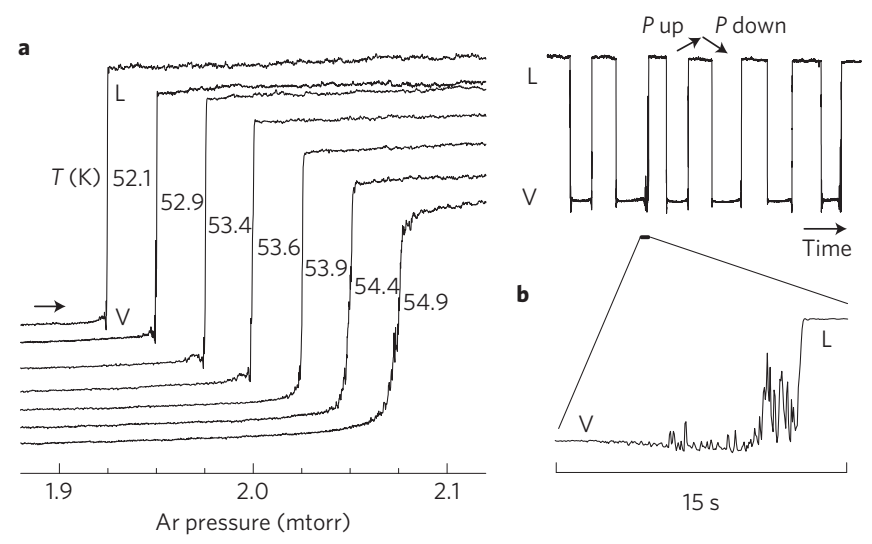

Figure 4 | Dynamical effects at a 2D phase transition. a, Conductance isotherms close to the $2 \mathrm{D}$ critical point, $T_{\mathrm{C}}=55.5 \mathrm{~K}$, for Ar on device YB14 Traces are individually offset along both axes for clarity; the pressure is

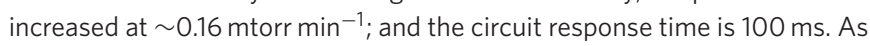
the riser becomes vertical, a sharp dip with associated temporal fluctuations appears at its foot. $\mathbf{b}$, Conductance monitored while gently increasing and decreasing the pressure at $51 \mathrm{~K}$ to repeatedly cross the $\mathrm{V}$ - $\mathrm{L}$ transition. Below is a zoom on one $\mathrm{V} \rightarrow \mathrm{L}$ jump.

pressures at the risers are shown on a $P-T$ diagram in Fig. 3b. They are higher than the pressures at the phase boundaries on graphite, indicated by dashed lines. Part of the difference is a systematic error of up to a factor of two caused by the cell pressure $P$ lagging the gauge pressure $P_{\mathrm{g}}$. The lower inset to Fig. 3a illustrates the hysteresis due to this lag at $51 \mathrm{~K}$, and how it can be reduced by slowing the sweep rate to home in on the true equilibrium pressure at the $\mathrm{L}-\mathrm{V}$ jump, which is plotted as an open red circle in Fig. 3 b. The remaining order-ofmagnitude pressure excess is due to weaker binding to nanotubes than to graphite ${ }^{1}$.

The conductance can also be employed to investigate critical behaviour. According to the theory of critical phenomena ${ }^{27}$ the compressibility of the monolayer, proportional ${ }^{11}$ to $\left(\phi^{2} T\right)^{-1} \mathrm{~d} \phi / \mathrm{d}(\ln P)$, should diverge approaching $T_{\mathrm{c}}$ in the supercritical regime as a negative power of the reduced temperature, $\tau \equiv\left(T-T_{\mathrm{c}}\right) / T_{\mathrm{c}}$. This behaviour cannot be studied in coverage measurements because the vibrational resonance is too distorted in this regime, but as $G$ is a smooth function of $\phi$ we anticipate the same scaling in the conductance isotherms. Figure $3 \mathrm{c}$ shows the maximum slope, $\mathrm{d} G / \mathrm{d}(\ln P)$, of each isotherm versus $T$. At $55 \mathrm{~K}$ and below, no matter how slowly we introduce gas, there is an instantaneous jump and the measurement is truncated by the 100 ms circuit response time. Inset is a $\log -\log$ plot of $(1 / T) \mathrm{d} G / \mathrm{d}(\ln P)$ versus $\tau$ taking $T_{\mathrm{c}}=55.5 \mathrm{~K}$ from the literature ${ }^{26}$. For larger $\tau$ it follows the power law $\tau^{-\gamma}$, where $\gamma=7 / 4$, the critical exponent for the $2 \mathrm{D}$ Ising model universality class which we expect to apply here. On the other hand the points at $\tau<0.03$ lie below the extrapolated power law, consistent with finite-size suppression of the power-law divergence ${ }^{28}$ roughly when $1 / \tau$ exceeds the nanotube a

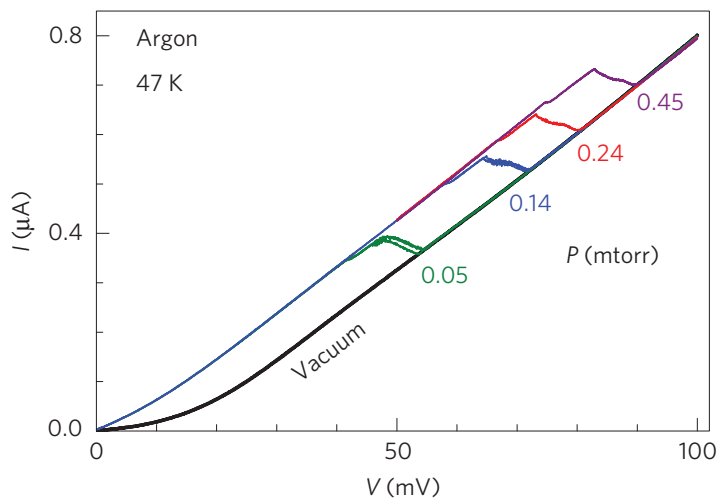

d

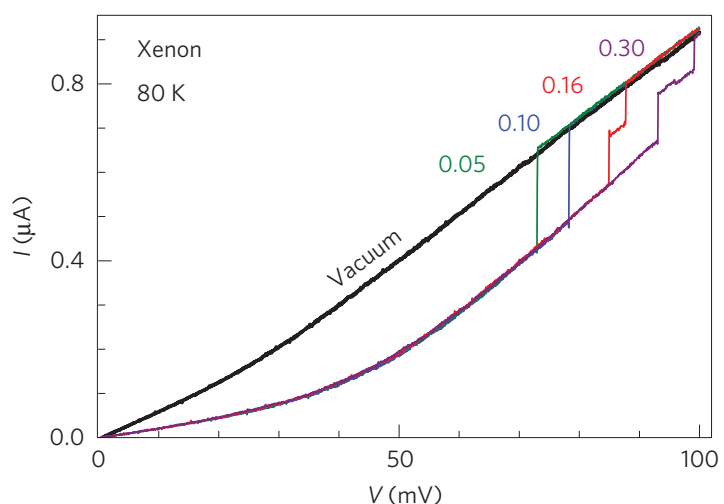

b

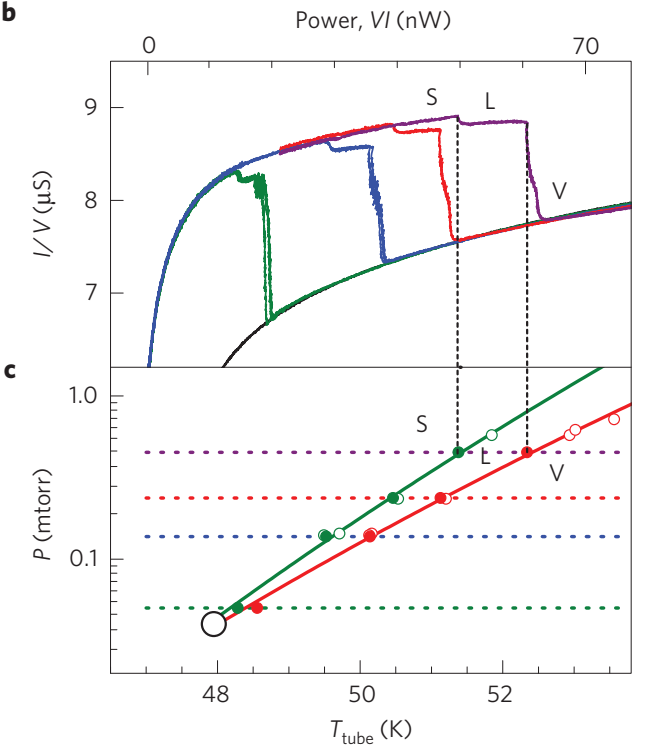

e

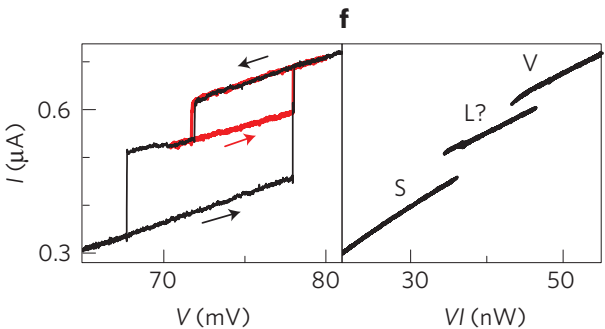

Figure 5 | Nonlinear behaviour. a, $I-V$ measurements on device $Y B 14$ in $A r$ vapour for several pressures at cell temperature $47 \mathrm{~K}\left(V_{\mathrm{g}}=0.34 \mathrm{~V}\right)$. Both up and down sweeps are plotted in each case. b. The same data plotted as conductance (I/V) versus power ( VI), showing near-vertical jumps linked to phase transitions in the Ar monolayer. c, The power at each jump is mapped to nanotube temperature (bottom axis; see text) and the positions of smaller steps are plotted as green solid circles, the larger steps as red solid circles. Open circles are from measurements at higher cell temperatures. The results lie close to the phase boundaries obtained from isotherms in Fig. 3. d, I-V measurements in Xe vapour at $80 \mathrm{~K}$ (here sweeping $V$ up only). e, Cycling $V$ reveals multiple metastable current levels $\left(P_{g}=0.16\right.$ mtorr). f, Same data plotted versus power, indicating $\mathrm{S}, \mathrm{V}$ and presumed $\mathrm{L}$ states. 
circumference divided by the diameter of an argon atom. This is the first experimental system to allow studies of critical behaviour in $2 \mathrm{D}$ with a periodic boundary condition.

The conductance also reveals the dynamics of the phase transitions. Figure $4 \mathrm{a}$ shows a series of closely spaced conductance isotherms near $T_{\mathrm{c}}$. Growing temporal fluctuations are seen on approaching $T_{c}$, where critical fluctuations are expected. They cannot be caused by sample temperature variations, which would also produce fluctuations away from $T_{\mathrm{c}}$. In addition, below $T_{\mathrm{c}}$ a sharp dip appears at the foot of the V-L step. A similar anomaly appears below $T_{\text {tr }}$ at the top of the V-S step (bold arrow), as can be seen in Fig. 3a, whereas none is seen at the top of the V-L step or on either side of the L-S step. These anomalies have large fluctuating components, as illustrated in Fig. $4 \mathrm{~b}$, where we monitor $G$ while nudging the pressure repeatedly up and down through the $\mathrm{L}-\mathrm{V}$ transition at $51 \mathrm{~K}$. In both directions the anomaly appears on the $\mathrm{V}$ side but not on the $\mathrm{L}$ side of the transition. The number of fluctuations recorded depends on the precise pressure sweep rate. An enlargement of a transition with many fluctuations is shown in the lower part of the figure.

Instead of using the conductance as a passive probe, we can pass a current large enough to perturb the adsorbates and produce nonlinear effects. Figure 5 a shows a set of $I-V$ sweeps, both up and down, measured in several pressures of Ar vapour at $47 \mathrm{~K}$. They exhibit a reproducible kink, with negative differential resistance, that moves to higher bias as $P$ increases. The kink is a transition between a higher conductance level, obtained when a monolayer is present, and a lower conductance level matching that obtained in vacuum (black). When the conductance $I / V$ is plotted against power VI (Fig. 5b) each kink becomes a nearly vertical jump, and another smaller jump is visible at lower power. The jumps can be attributed to phase transitions resulting from Joule heating of the nanotube above the cell temperature. Assuming the nanotube temperature $T_{\text {tube }}$ is uniform (justified because the heat is generated mainly at the contacts) and its rise is proportional to the power, we expect $T_{\text {tube }}=T+\alpha V I$, where $\alpha$ is a constant. By requiring consistency between measurements at different cell temperatures we derive $\alpha=0.088 \mathrm{KnW}^{-1}$. In Fig. $5 \mathrm{c}$ we plot the jump positions in terms of $T_{\text {tube }}$ at each pressure. The small jumps (green circles) and the larger jumps (red circles) lie on curves close to the S-L and $\mathrm{L}-\mathrm{V}$ boundaries, respectively, in Fig. 3b. We conclude that $I-V$ measurements can be used as isobaric temperature sweeps for rapidly mapping the phase diagram.

We see a variety of other nonlinear phenomena. For example, Fig. $5 \mathrm{~d}$ shows $I-V$ sweeps at $80 \mathrm{~K}$ in Xe vapour $\left(T_{\text {tr }}=99 \mathrm{~K}\right)$, which exhibit sharp jumps. On cycling $V$ there is hysteresis and metastability (Fig. 5e), a natural consequence of positive feedback because in this case the conductance, and thus heating, increases when the monolayer evaporates. However, the current is not a single-valued function of power (Fig. 5f), and the middle of the three levels has additional structure and a large difference in current from the low-bias $S$ state, hard to reconcile with a simple uniform equilibrium $\mathrm{L}$ state. There are also anomalies near the jumps reminiscent of those seen in the conductance isotherms, and other unexplained features including the sub-steps at $I / V \approx 8 \mu \mathrm{S}$ in the large jumps in Fig. 5b. Further studies using other adsorbates and nanotubes of known chirality will be needed to reveal the nature of such phenomena and to build a full understanding of coupling between adsorbates and conduction electrons.

\section{Methods}

Methods and any associated references are available in the online version of the paper.

Received 14 October 2014; accepted 4 March 2015; published online 20 April 2015

\section{References}

1. Stan, G. \& Cole, M. W. Low coverage adsorption in cylindrical pores. Surf. Sci. 395, 280-291 (1998).

2. Talapatra, S. \& Migone, A. D. Existence of novel quasi-one-dimensional phases of atoms adsorbed on the exterior surface of close-ended single wall nanotube bundles. Phys. Rev. Lett. 87, 206106 (2001).

3. Bienfait, M. et al. Thermodynamics and structure of hydrogen, methane, argon, oxygen, and carbon dioxide adsorbed on single-wall carbon nanotube bundles. Phys. Rev. B 70, 035410 (2004).

4. Kim, H. Y., Cole, M. W., Mbaye, M. \& Gatica, S. M. Phase behavior of Ar and Kr films on carbon nanotubes. J. Phys. Chem. A 115, 7249-7257 (2011).

5. Lee, H. C. et al. $\mathrm{Kr}$ and $\mathrm{He}-4$ adsorption on individual suspended single-walled carbon nanotubes. J. Low Temp. Phys. 169, 338-349 (2012).

6. Wang, Z. H. et al. Phase transitions of adsorbed atoms on the surface of a carbon nanotube. Science 327, 552-555 (2010).

7. Tavernarakis, A. et al. Atomic monolayer deposition on the surface of nanotube mechanical resonators. Phys. Rev. Lett. 112, 196103 (2014).

8. Chiu, H. Y., Hung, P., Postma, H. W. C. \& Bockrath, M. Atomic-scale mass sensing using carbon nanotube resonators. Nano Lett. 8, 4342-4346 (2008).

9. Jensen, K., Kim, K. \& Zettl, A. An atomic-resolution nanomechanical mass sensor. Nature Nanotechnol. 3, 533-537 (2008).

10. Chaste, J. et al. A nanomechanical mass sensor with yoctogram resolution. Nature Nanotechnol. 7, 300-303 (2012).

11. Dash, J. G. Films on Solid Surfaces (Academic Press, 1975).

12. Bruch, L. W., Cole, M. W. \& Zaremba, E. Physical Adsorption: Forces and Phenomena (Oxford Univ. Press, 1997).

13. Peng, S. \& Cho, K. J. Chemical control of nanotube electronics. Nanotechnology 11, 57-60 (2000).

14. Zhao, J. J., Buldum, A., Han, J. \& Lu, J. P. Gas molecule adsorption in carbon nanotubes and nanotube bundles. Nanotechnology 13, 195-200 (2002).

15. Leenaerts, O., Partoens, B. \& Peeters, F. M. Adsorption of $\mathrm{H}_{2} \mathrm{O}, \mathrm{NH}_{3}, \mathrm{CO}, \mathrm{NO}_{2}$, and NO on graphene: A first-principles study. Phys. Rev. B 77, 125416 (2008).

16. Cao, J., Wang, Q. \& Dai, H. Electron transport in very clean, as-grown suspended carbon nanotubes. Nature Mater. 4, 745-749 (2005).

17. Collins, P. G., Bradley, K., Ishigami, M. \& Zettl, A. Extreme oxygen sensitivity of electronic properties of carbon nanotubes. Science 287, 1801-1804 (2000)

18. Kong, J. et al. Nanotube molecular wires as chemical sensors. Science 287, $622-625(2000)$

19. Someya, T., Small, J., Kim, P., Nuckolls, C. \& Yardley, J. T. Alcohol vapor sensors based on single-walled carbon nanotube field effect transistors. Nano Lett. 3, 877-881 (2003).

20. Romero, H. E., Bolton, K., Rosen, A. \& Eklund, P. C. Atom collision-induced resistivity of carbon nanotubes. Science 307, 89-93 (2005).

21. Schedin, F. et al. Detection of individual gas molecules adsorbed on graphene. Nature Mater. 6, 652-655 (2007).

22. Wehling, T. O. et al. Molecular doping of graphene. Nano Lett. 8 , 173-177 (2008)

23. Robinson, J. A., Snow, E. S., Badescu, S. C., Reinecke, T. L. \& Perkins, F. K. Role of defects in single-walled carbon nanotube chemical sensors. Nano Lett. 6, 1747-1751 (2006)

24. Dan, Y. P., Lu, Y., Kybert, N. J., Luo, Z. T. \& Johnson, A. T. C. Intrinsic response of graphene vapor sensors. Nano Lett. 9, 1472-1475 (2009).

25. Millot, F. Adsorption of the 1st layer of argon on graphite. J. Physique Lett. 40, L9-L10 (1979).

26. Migone, A. D., Li, Z. R. \& Chan, M. H. W. Melting transition of submonolayer Ar adsorbed on graphite. Phys. Rev. Lett. 53, 810-813 (1984).

27. Yeomans, J. M. Statistical Mechanics of Phase Transitions (Oxford Univ. Press, 1992).

28. Auyang, H. \& Fisher, M. E. Bounded and inhomogeneous Ising models. 2. Specific-heat scaling function for a strip. Phys. Rev. B 11, 3469-3487 (1975).

\section{Acknowledgements}

This work was supported by NSF DMR award 1206208. Silicon structures were fabricated in the UCSB Nanofabrication Facility and the UW NTUF. We thank R. Roy for contributions in setting up the experiment, and M. den Nijs for helpful discussions.

\section{Author contributions}

D.H.C. and O.E.V. designed the experiments, B.D. and H-C.L. made the devices, all authors participated in measurements and analysis, and D.H.C. drafted the manuscript.

\section{Additional information}

Supplementary information is available in the online version of the paper. Reprints and permissions information is available online at www.nature.com/reprints. Correspondence and requests for materials should be addressed to D.H.C.

\section{Competing financial interests}

The authors declare no competing financial interests. 


\section{Methods}

The nanotubes are grown by chemical vapour deposition using $\mathrm{H}_{2} / \mathrm{CH}_{4}$ at $800^{\circ} \mathrm{C}$ and $\mathrm{Fe}\left(\mathrm{NO}_{3}\right)_{3} / \mathrm{MoO}_{2}$ catalyst, across trenches between pre-patterned Pt source and drain electrodes ${ }^{16}$ on $\mathrm{Si}_{3} \mathrm{~N}_{4} / \mathrm{SiO}_{2}$. The trenches are $1 \mu \mathrm{m}$ wide and $0.5 \mu \mathrm{m}$ deep, with a Pt gate on the trench bottom. We select nanotubes with small gaps

$(20-100 \mathrm{meV})$ because they have better electrical contacts than large-gap ones. The vapour cell is mounted in a closed-cycle cryostat with temperature range 4-300 K. The gas handling manifold and ceramic capacitance pressure gauge are at room temperature. The cell pressure $P$ is lower than the gauge pressure $P_{\mathrm{g}}$ in static equilibrium owing to thermal transpiration and is inferred by applying a correction $^{29}$. The linear-response conductance $G$ is measured using a $1 \mathrm{mV}$ a.c. source bias at $600 \mathrm{~Hz}$, a virtual-earth current preamplifier and a lock-in amplifier. The nonlinear $I-V$ is measured using a d.c. source bias. The coverage $\phi$ is measured by adding to the source bias a swept radio-frequency signal at frequency $f$ amplitude-modulated at $f_{\mathrm{am}}=1 \mathrm{kHz}$ (see Supplementary Information).

Mechanical resonances ${ }^{30}$ are identified as peaks in the mixing current measured by a lock-in amplifier referenced to $f_{\text {am }}$. The equation

$\phi=N_{\mathrm{a}} / N_{\mathrm{C}}=\left(m_{\mathrm{C}} / m_{\mathrm{a}}\right)\left[\left(f_{0} / f_{\mathrm{p}}\right)^{2}-1\right]$ gives a good measure of the coverage $\phi$, where $f_{\mathrm{P}}$ and $f_{0}$ are the positions of the mechanical resonance at pressure $P$ and in vacuum respectively, $m_{\mathrm{a}}$ is the molecular mass of the adsorbate, and $m_{\mathrm{C}}$ is the atomic mass of carbon ${ }^{6}$.

\section{References}

29. Takaishi, T. \& Sensui, Y. Thermal transpiration effect of hydrogen, rare gases and methane. Trans. Faraday Soc. 59, 2503-2514 (1963).

30. Sazonova, V. et al. A tunable carbon nanotube electromechanical oscillator. Nature 431, 284-287 (2004). 